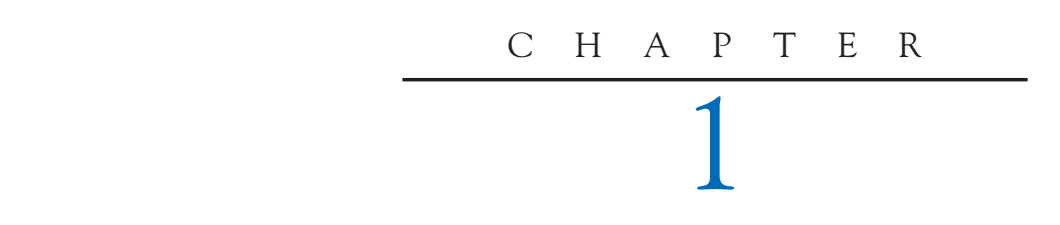

\title{
Setting up a Cell Culture Laboratory
}

\author{
Robert O'Connor and Lorraine O'Driscoll
}

\section{INTRODUCTION}

Over the past three decades, the continuous culture of eukaryotic cells has become a mainstay technique in many different forms of biological, biochemical, and biomedical experimentation. While at first, to the uninitiated, the techniques, methodology, and equipment can appear daunting, clear specification of the experimental requirements can help make choices straightforward. This article does not purport to be an exhaustive guide but rather aims to prompt the researcher to plan and make choices appropriate to their experimental, environmental, and financial resources.

\section{WHERE TO START}

Cell culture needs a commitment of energy and resources to be undertaken in a professional manner for any continuous period. Therefore, the biggest decision to be made before going down this experimental road is whether there will be an ongoing need for culture facilities or whether for short periods of work it might be more economical to collaborate with an established laboratory or sub contract work. Assuming that there is an agreed need to set up a cell culture facility, there are several fundamental considerations.

\section{A. Environment}

Purpose-built facilities are optimal for ergonomic and experimental reasons but often this choice is not available. Small cell culture facilities can be engineered (with inherent limitations) without making significant modifications to laboratory rooms. Before going into the detailed choices available, it is perhaps timely to go through some of the basics.

There are two fundamental considerations that govern most choices available to the would-be cell culture researcher: contamination and safety.

The fundamentals of cell culture owe much to the basic methodologies developed by microbiologists over the last two centuries. However, microorganisms reproduce several orders of magnitude more rapidly than eukaryotic cells and, in direct competition, bacteria and fungi will rapidly reproduce more biomass than eukaryotic cells. Eukaryotic cells are also very sensitive to the primary and secondary metabolic products of microbes. Bacteria and fungi are therefore the biggest problem, for those growing eukaryotic cells. As mentioned in the article by Meleady and $\mathrm{O}^{\prime}$ Connor, there is also potential for cross contamination of one cell line by another if proper procedures are not observed (a major problem with the first cultured human cell lines).

Eukaryotic cells can potentially harbour subcellular microbes that could cause disease to human beings or animals. More specifically, most human cell cultures are derived from human cancers. Being derived from human beings who could be harbouring several known (and potentially, as yet, unknown) pathogens, appropriate steps must be taken to ensure that cells do not pose a risk of passing disease on to human beings, including the researcher or others including visitors or cleaning staff. In practice, the majority of pathogenic organisms are quite fragile and do not survive well under general culture conditions. The risk of disease is therefore greatest when working with primary biological material, i.e., material recently removed from 
another (human) being. However, one should assume that any eukaryotic cells could potentially harbour microbes and/or viruses (including oncogenic viruses) and/or prion-contaminated material. As a general rule, taking the maximum amount of reasonable precautions (in procedures and equipment) gives the best margin of protection for staff, provides peace of mind for operators, and limits the culpability of supervisors/managers. The Centers for Disease Control (CDC) in the United States stipulate that general cell cultures should be undertaken in biosafety level 2 containment facilities (CDC, 1999).

\section{B. Location}

Having briefly outlined these fundamentals, it should be clear that correctly locating a cell culture facilities is of paramount importance. The working environment needs to be clean, free from dust, and easy to disinfect. The immediate area should have limited/restricted access, with no passing traffic. Consideration should be given to proper ergonomics in the area, e.g., correct heights of equipment, nothing requiring bending down, suitable chairs, and so on, to reduce the chance of chronic or acute injuries to staff (see the excellent laboratory website by the NIH for further details, specifications, and illustrations). Thought should also be given to how large and often heavy equipment, particularly laminar flow cabinets, can be brought in and out without major disruptions. If a building is being planned, this may include provisions for large lifts with fully opening doors or direct door access to higher floors with high loading equipment. Door openings need to be extra wide, typically at least $1 \mathrm{~m}$ full clearance, and corners designed that large equipment can get by. Provision must also be made for the movement and storage of consumables and bulk items. In practice, it is usually better to pool certain sets of equipment together in individual rooms, e.g., several biosafety cabinets being located with an incubator and other ancillary equipment. This reduces the overall equipment/cost necessary and can also make for a more "communal" working environment. Quarantine areas are an obvious exception to this suggestion.

\section{Gases}

Many cell cultures can be maintained in HEPESbuffered medium, which utilises carefully controlled incubators that do not need a separate carbon dioxide gas supply. However, some cells do not grow optimally in HEPES and need the buffering provided by the equilibrium of $5 \% \quad \mathrm{CO}_{2}$ with bicarbonate in medium. This can be important for some specific cell lines, primary culture, and hybridoma work (Freshney, 2000). At a minimum, such incubators should have two separate $\mathrm{CO}_{2}$ sources supplying them. Direct connection of a single cylinder means that the incubators are vulnerable every time a cylinder is changed and cylinders may fail to provide an adequate pressure of gas as they near an empty state. Cylinder changeover units permit, for example, one main line of gas and one backup cylinder, which means that incubators can be left for weeks or months (depending on use) without needing gas replacement and, when cylinders are replaced, there is no interruption in supply to the incubator (see NTC services website). Cylinders in a laboratory environment must always be fully fixed to an immovable object to lessen the risk of them toppling and doing serious injury and damage. Appropriate automatic changeover units can also be incorporated into external supplies of $\mathrm{CO}_{2}$. It is always better not to have large cylinders of gas in a culture room for safety, practical, and aesthetic reasons. Building regulations in some areas may also legislate against the use of large cylinders in enclosed rooms.

\section{Ventilation}

Ventilation and airflow in the cell culture environment are critical to operation. At its simplest, there must be no disruption to the laminar airflow pattern in the biological safety cabinet and no undue circulation of dust and dirt that could occur with, for example, significant staff movement around the unit or location near drafts or vents. Ideally, there should be no openable windows; if so, they should be sealed to prevent drafts, insects, and dust entering (Freshney, 2000). However, in a purpose-built facility, if possible, it is ergonomically and aesthetically desirable that there be a source of natural light. When planning, one should also make provisions in case there is a need to fumigate the room or equipment (Doyle, 1998). Cabinets will usually need to be fumigated in advance of any filter changes, although this can now usually be performed on single cabinets (using a cabinet bag system) rather than whole rooms.

Clearly, whatever the room design, there must be some replenishment of air in a room and such ventilation must not interfere with the operation of the cabinet. If liquid nitrogen is being utilised in the same location as the cabinet, the ventilation must be adequate to remove the continuous evaporation of liquid and the ventilation/air space sufficient to ensure that if there is an acute spillage of liquid or a rupture of the vacuum vessel, there will still be sufficient oxygen concentration to support life and permit evacuation, i.e., that instant evaporation of all the liquid nitrogen 
will not reduce the oxygen concentration below $14 \%$. If this cannot be guaranteed at all times, oxygen monitoring and alarm equipment will be required (Angerman, 1999).

High efficiency particulate air (HEPA) filtration within the safety cabinet will ensure a good quality working environment. However, if the air around the unit is dusty, the cabinet filters will age and clog quite rapidly. HEPA filtration is a statistical process (99.999\%-99.97\% efficient depending on the manufacturer) and unduly contaminated air may also reduce the air quality inside the cabinet. HEPA filtration of air into a cell culture room should improve operation and cabinet filter longevity and is also a requirement of biocontainment-classified rooms. However, if the room itself is not maintained properly, expensively ducted HEPA-filtered air may merely be clean air being utilised to circulate dust and microbes. Depending on the biocontainment level required for operation, HEPA filtration may also be required on exhaust vents for a cell culture room (class $2+$ biocontainment and above).

In larger cell culture facilities, careful balancing of air pressures in culture and anterooms may be useful or may be required for operation to appropriate biocontainment levels, specifically the positive and negative air pressures required for class 2 and greater biocontainment (CDC, 1999). Balancing and filtration require careful installation and validation from the outset and continued regular maintenance and monitoring to ensure appropriate function. Ventilation systems will also need to be tied into building fire management systems with, for example, automatic smoke dampeners to prevent the ventilation system from fanning or spreading smoke and fire.

\section{E. Basic Cell Culture Requirements}

To perform a basic range of cell culture procedures for any prolonged period the following list of equipment is required (Freshney, 2000).

\author{
Laminar flow cabinet \\ Incubator \\ Centrifuge \\ Refrigerator \\ Freezer \\ Microscope \\ Haemocytometer \\ Pipette boy \\ Micropipette
}

Outside immediate culture environment
Autoclave
Selection of appropriate consumables and
cultureware

General laboratory facilities for the storage of chemicals, provision of standard reagents such as clean water, and controlled temperature water baths

\section{F. Ideal Layout}

\section{Purpose-Built Facility}

There are significant advantages in having the resources to custom design and implement a purposebuilt facility. A purpose-built facility should be strongly considered (and will likely be more economical in the long term) if cell culture is performed on a significant scale (involving many researchers) for an established organisation and/or if this work is likely to be undertaken on a commercial basis. Aside from the support departments, which are mentioned later, the standard accepted laboratory scheme for cell culture involves a general laboratory room, an anteroom (physically and methologically separating the general laboratory area from the cell culture room), and a specific, self contained, cell culture room.

\section{Equipment}

a. Biosafety Cabinets. While basic cell culture can be performed with sterile equipment and good techniques, there is no question that the development of modern biological cabinets has greatly facilitated routine culture procedures and the prolonged manipulation of cells required for many experimental procedures. Laminar flow cabinets come in many sizes, capacities, and with several variants appropriate for different types of cell culture. Most manufacturers have excellent technical schematics describing the appropriate operation, dimensions, and so on. For examples, see the websites by Heto-Holten or Baker. Any laminar flow cabinet should be designed to an internationally recognised specification and biological safety standard appropriate to the work being undertaken or likely to be performed in at least the next 5 years. [See CDC $(1995,2000)$ and the list of international specifications in the Appendix for further details.] Laminar flow cabinets represent a significant investment and will usually give decades of service if adequately maintained. A careful choice is therefore important. The precise operation of a laminar flow cabinet is beyond the scope of this review except to say that they fundamentally consist of a large air-pumping motor, pumping air through a HEPA filter onto a working surface (CDC, 2000). Cabinet HEPA filters are designed to remove particulates (from proportionately large dust down to submicroscopic viral particles). Forcing large volumes of air through these filters needs large motors, which inherently imply significant 
weight in their own right, and a heavy chassis to support this weight and to prevent vibration. Before locating/installing, one therefore needs to consider how the unit is to be placed in the required position. Typical weights can vary from $200 \mathrm{~kg}$ for new models to an excess of $500 \mathrm{~kg}$ for older units (which may still be very usable). More modern concrete-fabricated buildings will usually be designed to take the weight of one or more cabinets on a limited floor space, but this may need to be checked with an appropriate engineer and will certainly cause limitations in many lighter prefabricated or older buildings. A clear path from the point where a delivery vehicle may drop off the cabinet to its final resting place is required. Doors will need to be wide enough for the delivery pallet or, at least, the unpacked unit. Lift size and weight restrictions may be a particular problem, as can sharp corners on the route. Lift specifications should always be in excess of the largest envisaged unit that they might be expected to transport. In addition or if an appropriate lift cannot be provided, direct access to corridors on upper floors should be provided so that low loaders or cranes can directly insert heavy equipment to each floor of the building. Although one should plan for the unit to be in place for many years, one should also consider possibilities of bringing in new units and/or removing units as a particular group of scientists may expand or move their operation. Careful planning is therefore essential. Some manufacturers also have built-in modifications or supply units in subsections, which allow their units to take up a smaller space during movement than required for the operational unit.

Which Unit to Purchase/Use? Several international bodies have developed standard specifications for laminar flow cabinets (see specification list in Appendix). General cell culture requires a class II cabinet for an adequate protection of cells and operator (see Section A). These units recirculate HEPA-filtered air and exhaust a portion of that air back into the room through a HEPA filter. Where hazardous agents may be used, higher specification units such as external venting cabinets may be required (e.g. class II type B2/B3). These require very specific and costly exhaust ducting (CDC, 2000).

A careful choice of cabinet size should be made. In our centre, many researchers prefer a 4 -ft $(1.2 \mathrm{~m})$-wide laminar with plenty of space between the inner top of the cabinet and the work surface. Smaller cabinets may suit more restricted spaces but also restrict the amount of work that can be undertaken in comfort inside. Larger units may be useful for bulkier operations such as batch media production. The operator should be able to get their legs fully under the working space to permit appropriate posture and to reduce stretching and bending, which can cause repetitive strain injuries. The unit and the culture room should be well lit with low flicker lighting of an appropriate intensity (preferably supplemented with natural light). The motor size of safety cabinets causes the units to give off a significant amount of heat. A very significant heat load may be generated in areas where there are several (nonducted) units in a confined space with incubators. The air-handling system must be able to cope with such a loading and maintain the temperature at a comfortable level. Optional extras, such as ultraviolet lights, are often dispensable and may have limitations or be inappropriate. If laboratory benches are used to support the cabinet, they must be sufficiently sturdy to support the full weight of the unit for the duration of use. Legs must also be sufficiently broadly spaced to prevent any risk of toppling.

b. Incubators. Most mammalian cells are maintained at $37^{\circ} \mathrm{C}$, whereas insect cell cultures typically grow at $28^{\circ} \mathrm{C}$. As mentioned previously, there may also be a need for a regulated use of $\mathrm{CO}_{2}$ and possibly other gas mixtures in the cabinet. Many different-sized incubators are available to suit the needs and requirements of the researcher. Temperature control and accuracy usually to $0.1^{\circ} \mathrm{C}$ is an obvious requirement, and units that have heated doors and glass inner doors, permitting limited observation without letting all the heat out, are preferable. In our laboratory, units of 200- to 300-liter internal capacity are capable of supporting the culture output of several researchers. Roller bottle and spinner flask culture vessels may also require special incubators or adapters. The incubator should ideally be located close to the laminar cabinet to reduce temperature changes, which could affect cultured cells. General cell culture incubators require the ambient temperature to be usually $5-10^{\circ} \mathrm{C}$ colder than the target temperature. If the ambient temperature is too close to the target temperature, standard units can overheat, making temperature control in the room critically important for reliable operation. The incubator should also be at standing height to prevent the need for bending down. Temperature-controlled warm rooms may be useful in specific circumstances; however, larger incubators and particularly warm rooms are very difficult to maintain at a homogeneous temperature all through the space, which can adversely affect growth.

All internal surfaces should be polished metal and accessible for cleaning purposes. Any surface or component that cannot be cleaned but is inside the air 
space of the incubator is likely to see significant microbial growth as residue builds up. Where vented culture flasks and $\mathrm{CO}_{2}$ are being used, the unit must also have a bath of clean water inside and the incubator must monitor relative humidity. Vented flasks and other unsealed culture vessels, such as 96-well culture plates, will rapidly dry out if humidity drops.

Most units available now are very straightforward to calibrate the level of $\mathrm{CO}_{2}$ and temperature. Sensors that use infrared measurement of $\mathrm{CO}_{2}$ are the easiest to calibrate as opposed to older electrochemical sensors. It is advisable to periodically check the calibration of such equipment using external measuring devices.

c. Centrifuges. Many different models of centrifuge are available for cell culture. For ergonomic reasons, the unit should be located near the biosafety cabinet and at an appropriate height. All centrifuges will vibrate to some degree, and measures should be taken to ensure that such vibrations cannot damage other equipment, cause unnecessary noise, or allow the centrifuge to creep and potentially fall. The centrifuging of tubes containing media and cells can induce aerosols, and all centrifuges used for cell culture and biological procedures must have some form of seal either above the rotor head or over the buckets, which prevents aerosol leaking, particularly where tubes may rupture or leak during operation. A common operational centrifugation rate is appproximately $110 \times g$, and units must capable of fractions and low multiples of this rate to cope with different research requirements. Temperature control, particularly the ability to spin at refrigerated temperatures, can be a very useful add on to standard units. The buckets in the rotor should have adapters that snugly fit the standard consumables used for culture in that facility, e.g., 25- and 50-ml universal tubes. Control of the acceleration and deceleration rates can also be useful when working with certain very sensitive cell lines.

d. Refrigerator/Freezer Units. Storage of media, media components, buffers, and so on needs refrigeration and freezing facilities. Domestic-type units are often used for this purpose; however, domestic units can have limitations in their ability to maintain a steady temperature and must also never be used if flammable liquids are to be stored inside (an externally thermostat-controlled unit must be used for such purposes). It can also be useful if the door has a lockable latch, as this can help ensure that the unit is closed properly after every use. In practice, careful monitoring of the storage and use of refrigerators/freezers are necessary to prevent space wastage. Fridges and freezers should be cleaned out regularly. When this is not done, it is common to see gradual increases in the number of such units, which can greatly add to heat loads in the laboratory. In larger scale facilities, it may be prudent to combine the use of fridges/freezers with longer term, volume storage in central cold/freezer rooms. In such laboratories it may also be useful to use cooling/freezing units that have condenser units located outside the laboratory. Although not usually required for cell culture operations, freezers that can operate below $-60^{\circ} \mathrm{C}$, usually $-80^{\circ} \mathrm{C}$, are often necessary for the storage of molecular biological enzymes and reagents.

e. Liquid Nitrogen. Maintenance of cultured cells for any prolonged period requires that cell stocks can be kept well below the glass temperature of water (approximately $-60^{\circ} \mathrm{C}$ ). In practice, liquid nitrogen storage is often a convenient general storage environment for stocks of cultured cells. However, electrical freezers operating at $-120^{\circ} \mathrm{C}$ can perform a similar function. Liquid nitrogen boils at $-196^{\circ} \mathrm{C}$, and because the resultant nitrogen gas is an asphyxiant in high concentrations, proper handling and ventilation procedures are necessary (see article by Meleady and $\mathrm{O}^{\prime}$ Connor). Proper inherently safe methods for transport and storage must be used to get the liquid gas into the cell storage containers. As the storage vessels usually require topping up at least once a week (more regularly as the containers age), the route from the gas delivery/production area to the laboratory must be as short as possible and the surface and equipment appropriate for the transport involved. Storage of cells by multiple users for prolonged periods necessitates careful inventory management to ensure that cells are maintained optimally and economically. It is good practice to have two independently stored stocks of cells: one vessel containing master stocks of important cells and the other, in the laboratory environment, containing working stocks.

Modern incubators, refrigerators, freezers, and liquid nitrogen vessels typically have alarms that can be set if parameters, such as temperature, drop below a critical values. However, such alarms are only of use if there is someone nearby to hear them. Where critical or expensive procedures are being utilised, it is wise to have such equipment linked into a monitoring system that can alert a researcher day or night. Consideration should also be given to the provision of a backup electricity generator that can automatically restore supply for a finite period in the event of an electrical blackout. 
f. Autoclave. Modern single-use plastic consumables have reduced the dependence on a laboratory autoclave for sterilising equipment and reagents; however, an appropriately sized, robust autoclave is still vital for continued cell culture work. Although appropriately sourced plastic consumables and presterilised reagents can eliminate the potential for contamination, there can still be huge cost savings by utilising specific pieces of glass equipment that can be repetitively autoclaved, used, and recycled, particularly reagent and media bottles, and by sterilising one's own general reagents, such as water and phosphate-buffered saline. Small autoclaves can generate significant amounts of foul odour, steam, and heat and should not be used in a general laboratory environment. Because of the biohazardous nature of cell culture, autoclaving of all materials and reagents that come into contact with cells is also required. For larger facilities, it is strongly recommended to have a centralised autoclave facility away from an individual laboratory. A distinction of clean and waste autoclaves is necessary to prevent cross contamination, particularly of odours and volatile substances. A backup autoclave is also good insurance against maintenance and unanticipated downtime. As such facility autoclaves are large, need regular maintenance and inspection, and can have a limited life span, provision for easy access and removal/replacement is advisable. The operational characteristics of the autoclave should also be regularly checked with spore strips, for example, to validate that the autoclave is operating effectively.

\section{General Environmental Recommendations}

Ideally, there should be a limited facility for the temporary storage of small amounts of culture consumables next to the biosafety cabinet. However, cardboard can be a significant source of fungal spores, and large-scale storage in the culture area causes clutter. Consumables are best stored in a central location, and if cell culture is being undertaken on a larger scale, it is far more economical to bulk purchase supplies and distribute them as necessary to each laboratory. Provision also needs to be made for the storage of flammable materials, especially disinfectant alcohols, which are used for local disinfection and "swabbing" (disinfectant wiping of consumables and reagents as they enter the working space inside safety cabinets).

Ideally, all flooring, walls, and other surfaces in the culture environment should be readily accessible, chemically resistant, nonadsorbent, and easy to clean. Shelves should not be used, and open flat surfaces should be minimised as these will need to be cleaned and can be a source for the buildup of dirt and dust.
Walls should be smooth skimmed and coated with epoxy paint. The floor should also be resistant and bonded to the wall so that there are no crevices or corners that cannot be cleaned and any spillages can be easily isolated and cleaned. Sinks, coat hooks, and so on should all be kept in an anteroom and not in the culture room.

Regular training, standard operating procedures, and centralised management of all aspects of cell culture, particularly technique and safety, ensure that there is an economical, continuously monitored, high standard of operation. The function of vital equipment should be continuously monitored and recorded. Particular attention should be paid to the monitoring of environmental microbial levels throughout the facility. For example, "settle" plates should be periodically left in laminar flow cabinets to check for sterility, as well as in all laboratory areas to validate the cleanliness of such areas.

The microbial quality of cell stocks should also be monitored as part of this process. All cells coming into a facility, regardless of the source, especially if supplied by noncommercial sources, should be quarantined and initially cultured in isolation from the general culture environment. Mycoplasma is the main microbial contaminant of concern because such contamination can go unnoticed for long periods and is very easy to pick up and cross contaminate stocks of cells. Ideally, a facility should have provision for routine Mycoplasma detection. Experience and expertise are required to do this job reliably, and where such expertise is not available in-house, commercial testing facilities exist.

Industrial disinfectants such as Virkon and/or Tego need to be employed (at recommended dilutions) as part of the routine cleaning of equipment and areas, in addition to diluted industrial methylated spirits or isopropyl alcohol, which is used for local disinfection and "swabbing."

Other Areas. A successful laboratory will have a two-way flow of people and materials. To manage and control these, it is useful to have a centrally located reception area to administer all incoming deliveries of consumables, cells, chemicals, and so on. Provision needs to made for effective communication within the organisation, including phone access in all but the most specialist of rooms, internet, and e-mail access and storage of records and scientific literature. If the unit is fully self-contained, the human environment must also be considered with an adequate provision of locker and toilet facilities and appropriate locations for taking breaks and eating completely away from the laboratory. 


\section{SUMMARY}

The reader will note the preference in this text for larger centralised, purpose-built facilities. In individual situations, it may be appropriate to represent the areas mentioned in a smaller more general way; however, even at its simplest, maintenance of a small cell culture environment necessitates that time be spent validating and monitoring the quality of that environment and the various flow paths contained therein. This obviously reduces the time available for research. However, in larger facilities, such critical validatory tasks can become specific jobs enabling the researcher to concentrate with confidence on biological research. Where such facilities are properly established, it is common that they continue to expand. The final thought in setting up a cell culture environment is to look as much as possible to the future and to allow for changes in use and facility expansion from the very start.

\section{APPENDIX}

The following relevant international standards are used for biosafety cabinets.

American National Standards Institute (National Sanitation Foundation). NSF/ANSI-49 (1992). Class II (Laminar Flow) Biohazard Cabinetry. Now superseded by NSF/ANSI49 (2002) and NSF/ANSI 49-02e (2002).

Australian standards AS2252.1, Biological Safety Cabinets (Class I) for personal protection (1980). AS2252.2 Biological Safety Cabinets (Class II) for personal protection (1981). Standards Association of Australia.

Canadian Standards Association, CSA Z316.3-95 (1995). Biological Containment Cabinets: Installation and Field Testing.

European standard EN12469:2000 (2000). Performance criteria for microbiological safety cabinets. Supersedes EU member state standards such as BS 5726 (British), DIN 12950 (German), and NF X44-201 (French).
Japanese Industrial Standard JIS K 3800 (JACA) for Class II biological safety cabinets.

South African standards, SABS 0226:2001 (2001). The installation, postinstallation tests, and maintenance of microbiological safety cabinets (2001) and VC 8041:2001, microbiological safety cabinets (Classes I, II, and III) (2001).

\section{References}

Angerman, D. (1999). "Handbook of Compressed Gases," 4th Ed. Kluwer Academic, New York.

Baker Biosafety cabinet website. http://bakercompany.com/ products/ 161 Gatehouse Road, Sanford, Maine 04073 USA.

CDC (1995). "Biosafety Cabinets; Primary Containment for Biohazards: Selection, Installation and Use of Biological Safety Cabinets," 1st Ed. U.S. Department of Health and Human Services Public Health Service Centers for Disease Control and Prevention and National Institutes of Health. U.S. Government Printing Office, Washington. Web edition http://www.niehs. nih.gov/odhsb/biosafe/bsc/bsc.htm

CDC (1999). U.S. Department of Health and Human Services Public Health Service Centers for Disease Control and Prevention and National Institutes of Health. "Biosafety in Microbiological and Biomedical Laboratories," 4th Ed. U.S. Government Printing Office, Washington. Web edition http://www.cdc.gov/ od/ohs/biosfty/bmbl4/bmbl4toc.htm. See also the CDC Office of Health and Safety biosafety general website: http://www.cdc.gov/od/ohs/biosfty/biosfty.htm

CDC (2000). "Primary Containment for Biohazards: Selection, Installation and Use of Biological Safety Cabinets." U.S. Department of Health and Human Services Public Health Service Centers for Disease Control and Prevention and National Institutes of Health. U.S. Government Printing Office, Washington. Web edition http://www.cdc.gov/od/ohs/biosfty/bsc/bsc.htm

Doyle, A. (1998). "Cell and Tissue Culture: Laboratory Procedures" (A. Doyle, J. B. Griffiths, and D. G. Newell, eds.), Chap. 1. Wiley, Chichester.

Freshney, R. I. (2000). “Culture of Animal Cells: A Manual of Basic Technique," 4th Ed. Wiley-Liss, New York.

Heto Holten Biosafety cabinet website. http://www.hetoholten.com/prod-holten.htm. Heto-Holten now part of Jouan Nordic, Gydevang 17-19, DK-3450, Allerød, Denmark.

National Institutes of Health (NIH) laboratory safety website http://www.nih.gov/od/ors/ds/ergonomics/lab3.html

NTC services limited. Northern Technical \& Chemical Services. Unit D44, Brunswick Business Centre, Liverpool, L3 4BD.UK. http://www.merseyworld.com/ntcs/ 